\title{
Cerebrolysin for functional recovery in patients with acute ischemic stroke: a meta-analysis of randomized controlled trials
}

This article was published in the following Dove Press journal:

Drug Design, Development and Therapy

19 April 2017

Number of times this article has been viewed

\author{
Zefeng Wang ${ }^{1, *}$ \\ Ligen Shi',* \\ Shenbin $X u^{\prime}$ \\ Jianmin Zhang ${ }^{1-3}$
}

'Department of Neurosurgery, Second Affiliated Hospital, School of Medicine, ${ }^{2} \mathrm{Brain}$ Research Institute, ${ }^{3}$ Collaborative Innovation Center for Brain Science, Zhejiang University, Hangzhou, People's Republic of China

*These authors contributed equally to this work
Correspondence: Jianmin Zhang Second Affiliated Hospital, School of Medicine, Zhejiang University, 88 Jiefang Road, Hangzhou 310009, Zhejiang, People's Republic of China

Tel +86 57। 8778 47। 5

Fax +86 57I 87784755

Email zjm 135@vip.sina.com
Abstract: Cerebrolysin has been shown to have an inconsistent efficacy on functional recovery in patients with acute ischemic stroke (AIS). The present meta-analysis aims to evaluate the value of cerebrolysin and to explore the potential influencing factors. The main electronic databases, including MEDLINE, EMBASE, and the Cochrane Library, were searched. The primary outcome was functional recovery at Day 90 . The secondary outcomes included mortality and adverse events. A total of 1,649 patients with AIS were pooled from six randomized controlled trials (RCTs). Cerebrolysin had no significant effect on functional recovery at Day 90 compared with the effect of placebo as shown by the modified Rankin Scale response (relative risk [RR] 1.33, 95\% confidence interval [CI] 0.79-2.24, $P=0.28$ ), National Institutes of Health Stroke Scale response (RR 1.03, 95\% CI 0.83-1.28, $P=0.77$ ), and Barthel Index response (RR $0.95,95 \%$ CI $0.84-1.08, P=0.44$ ). In safety analysis, cerebrolysin did not increase the risk of adverse events (RR $0.98,95 \%$ CI $0.88-1.09, P=0.67$ ), risk of serious adverse events (RR $1.20,95 \%$ CI $0.86-1.66, P=0.29$ ), or the mortality rate (RR $0.86,95 \%$ CI $0.57-1.31$, $P=0.49$ ). In conclusion, routine administration of cerebrolysin to patients with AIS cannot be supported by the available evidence from RCTs.

Keywords: acute ischemic stroke, cerebrolysin, functional recovery, meta-analysis, randomized controlled trials

\section{Introduction}

Acute ischemic stroke (AIS) is the second leading cause of death worldwide and the third most common cause of substantial disability, which contributes largely to the financial burdens of society. ${ }^{1}$ Intravenous thrombolysis and mechanical thrombectomy within 4.5-6 hours of stroke have been shown to significantly reduce the mortality rate and improve clinical outcomes. ${ }^{2,3}$ However, only $<20 \%$ of all AIS patients benefit from these reperfusion therapies. ${ }^{4}$ Poststroke neurological deficit is the most important factor influencing quality of life of AIS patients. ${ }^{5}$ There is an unmet need for neuroprotective or neurotrophic drugs with good efficacy in neurological functional recovery in AIS patients.

Cerebrolysin, a neuropeptide preparation of porcine origin, consists of low-molecularweight neuropeptides and free amino acids. ${ }^{6}$ Preclinical studies have indicated that cerebrolysin has neuroprotective properties and neurotrophic activity. ${ }^{6}$ In experimental ischemic stroke studies, cerebrolysin has been shown to reduce the infarction volume and improve functional recovery by inhibiting free radical formation, ${ }^{7}$ protecting against excitotoxicity, ${ }^{8}$ decreasing neuroinflammation and apoptosis, ${ }^{9}$ promoting neuronal sprouting, ${ }^{10}$ improving cellular survival, ${ }^{7}$ and stimulating neurogenesis. ${ }^{11}$ In addition, (c)
hereby accept the Terms. Non-commercial uses of the work are permitted without any further permission from Dove Medical Press Limited, provided the work is properly attributed. For permission for commercial use of this work, please see paragraphs 4.2 and 5 of our Terms (https://www.dovepress.com/terms.php). 
cerebrolysin has been shown to significantly improve neurological functional recovery in the subacute stages of AIS, that is, $\leq 48$ hours after onset of symptoms. ${ }^{11,12}$ This inspiring efficacy and the wide therapy window prompted an opportunity for clinical studies. Several clinical randomized controlled trials (RCTs) have been performed to examine the effect of cerebrolysin on neurological functional recovery in AIS patients. ${ }^{13-18}$ However, these trials have had inconsistent outcomes. Treatment with cerebrolysin showed no significant improvement in global status as assessed using the Canadian Neurological Scale, ${ }^{13}$ Barthel Index (BI), ${ }^{13-15}$ Clinical Global Impressions scale, ${ }^{13}$ modified Rankin Scale (mRS), ${ }^{14,15,18}$ National Institutes of Health Stroke Scale (NIHSS), ${ }^{14,15,18}$ and ten other different outcome scales in four RCTs. ${ }^{18}$ However, the Amiri-Nikpour et al trial showed a significant improvement in the NIHSS. ${ }^{16}$ These confusing outcomes might be due to limitations in sample size, the selection of the included patients, and the therapeutic regimen. Three RCTs indicated a superiority of cerebrolysin compared with placebo in a subgroup of AIS patients with severe neurological deficits at baseline. ${ }^{14,17,18}$ Two recent RCTs applied several specific neurological outcome scales to examine the effect of cerebrolysin on motor functional recovery and observed inconsistent outcomes. ${ }^{17,18}$ Hence, the present meta-analysis aims to evaluate the value of cerebrolysin and to explore the potential influencing factors.

\section{Methods}

At the onset of this project, a study protocol was drafted following the Preferred Reporting Items for Systematic Reviews and Meta-Analyses format guidelines. ${ }^{19}$ Our data were obtained from previous studies, and therefore, there was no need for approval from an ethics board.

\section{Search strategy and information sources}

Two investigators (ZFW and LGS) independently searched major electronic databases, including MEDLINE, EMBASE, and the Cochrane Library, for relevant studies published from January 1980 to May 2016. The combinations of the variables "cerebrolysin" AND "ischemic stroke" were used to match the titles and abstracts in the MEDLINE database. The search strategy for EMBASE and the Cochrane Library were similar to that for MEDLINE. In addition, two investigators (ZFW and LGS) also independently screened reference lists from RCTs, comments, meta-analyses, and reviews to ensure that all relevant studies had been included in this study.

\section{Study selection and data collection}

Only RCTs with AIS patients receiving cerebrolysin for functional recovery were applicable. The eligibility criteria were as follows: studies should have included a) adult subjects (aged $>18$ years), b) follow-up for 3 months after treatment, c) patients receiving intravenous cerebrolysin, and d) end points such as functional recovery.

All records were independently evaluated by two authors (ZFW and LGS) in accordance with the eligibility criteria as mentioned above. The following data were extracted from the included RCTs after strict selection and evaluation: information regarding the included trials, eligibility criteria and study design, and outcome assessments (Table 1).

\section{Outcome definitions and quality assessment}

The primary efficacy end point was a favorable outcome of functional recovery, which was defined as an $\mathrm{mRS}$ response (score $0-1$ ), NIHSS response (score $\geq 8$ from baseline or score $\leq 1$ ), or BI response (score 75-100) at Day 90. Secondary outcomes included mortality, adverse events, and serious adverse events at Day 90. The definition of serious adverse events was any life-threatening events, including acute coronary syndrome, atrial fibrillation, cardiac arrest, cardiac failure, sepsis, coma, renal failure, respiratory failure, cerebral hemorrhage, pulmonary embolism, and so on.

The risk of bias for all of the included RCTs was independently assessed by two investigators (ZFW and LGS) and evaluated with Cochrane Collaboration's risk-of-bias tool. ${ }^{20}$ Any differences were clarified and confirmed by a third assessor (JMZ) when possible. The risk-of-bias criteria evaluated the adequate generation of a randomization sequence, allocation concealment, blinding of participants, blinding of assessment, and dealing with missing data. For each item, the table provides a description and judgment rated as "low", "unclear", or "high" risk of bias. The risk-of-bias plot was created using the Review Manager 5.2 software.

\section{Data synthesis and analysis}

All data analyses were performed by two investigators (ZFW and SBX) using Review Manager 5.2 software. The relative risk (RR) and 95\% confidence interval (CI) were used as the effect measures of dichotomous outcomes in the metaanalyses. Heterogeneity in variance across studies was tested with the $Q$ and $I^{2}$ statistic. A significant $Q$ statistic indicates that the individual effect sizes do not represent a common population mean and that the effect size is considerably heterogeneous. A larger $I^{2}$ statistic also indicates a higher probability of heterogeneity. We used random-effect models (DerSimonian and Laird method) throughout as we found significant heterogeneity in our analyses. When heterogeneity is present, random-effect models are a more 


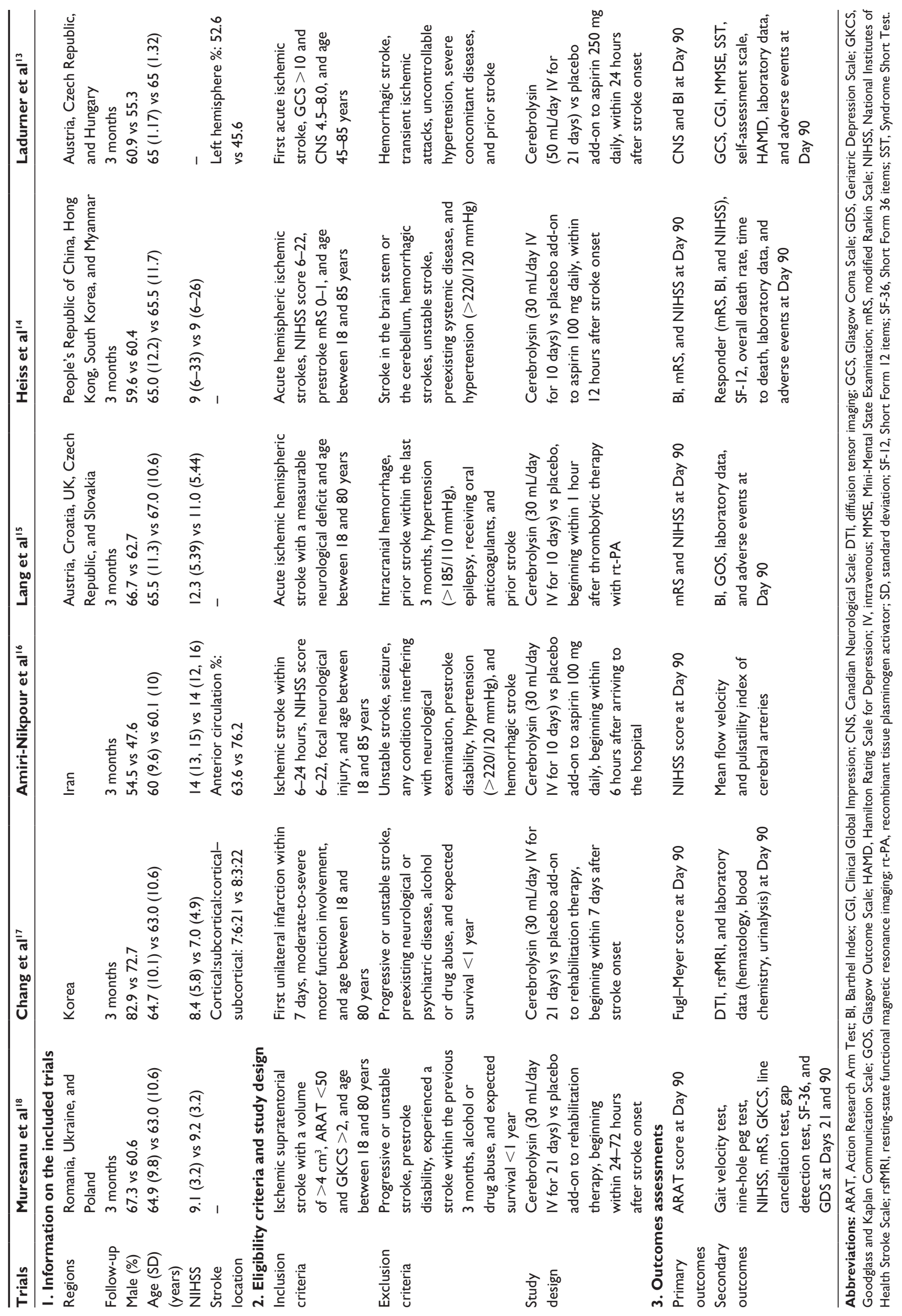


appropriate option for computations as they are less likely to reject the null hypothesis making them a more conservative estimate. Subsequently, random-effect models are more robust to large variations in sample size. In addition, we investigated the heterogeneity through a sensitivity analysis of the effect of omitting each study in turn. This method assesses whether the inclusion of any one study systematically affects the overall findings, allowing for the inclusion of methodologically flawed studies if they meet this criterion. Tests were two-tailed, and a $P$-value of less than 0.05 was considered to be significant for all analyses.

\section{Results}

\section{Study selection and characteristics}

A total of 161 titles and abstracts were screened (Figure 1). After removing the duplicates and irrelevant records, 26 full-text articles were assessed for eligibility. Twenty articles were further excluded due to the limitation of the publication types: one protocol study, three systematic reviews, two comments, six retrospective studies, and eight reviews. Ultimately, we identified six RCTs for meta-analysis. ${ }^{13-18}$ All of the included RCTs were international multicenter trials except for two studies. ${ }^{16,17}$ The detailed characteristics of the included studies are listed in Table 1.

\section{Efficacy and safety end points}

A total of 1,649 patients with AIS were pooled from the six RCTs. As different neurologic outcome scales were applied in the included studies, only four trials provided appropriate data for primary efficacy analysis. Cerebrolysin had no significant effect on functional recovery at Day 90 compared with the effect of placebo as shown by the mRS response (RR 1.33, 95\% CI 0.79-2.24, $P=0.28$; Figure 2A), NIHSS response (RR 1.03, 95\% CI 0.83-1.28, $P=0.77$;

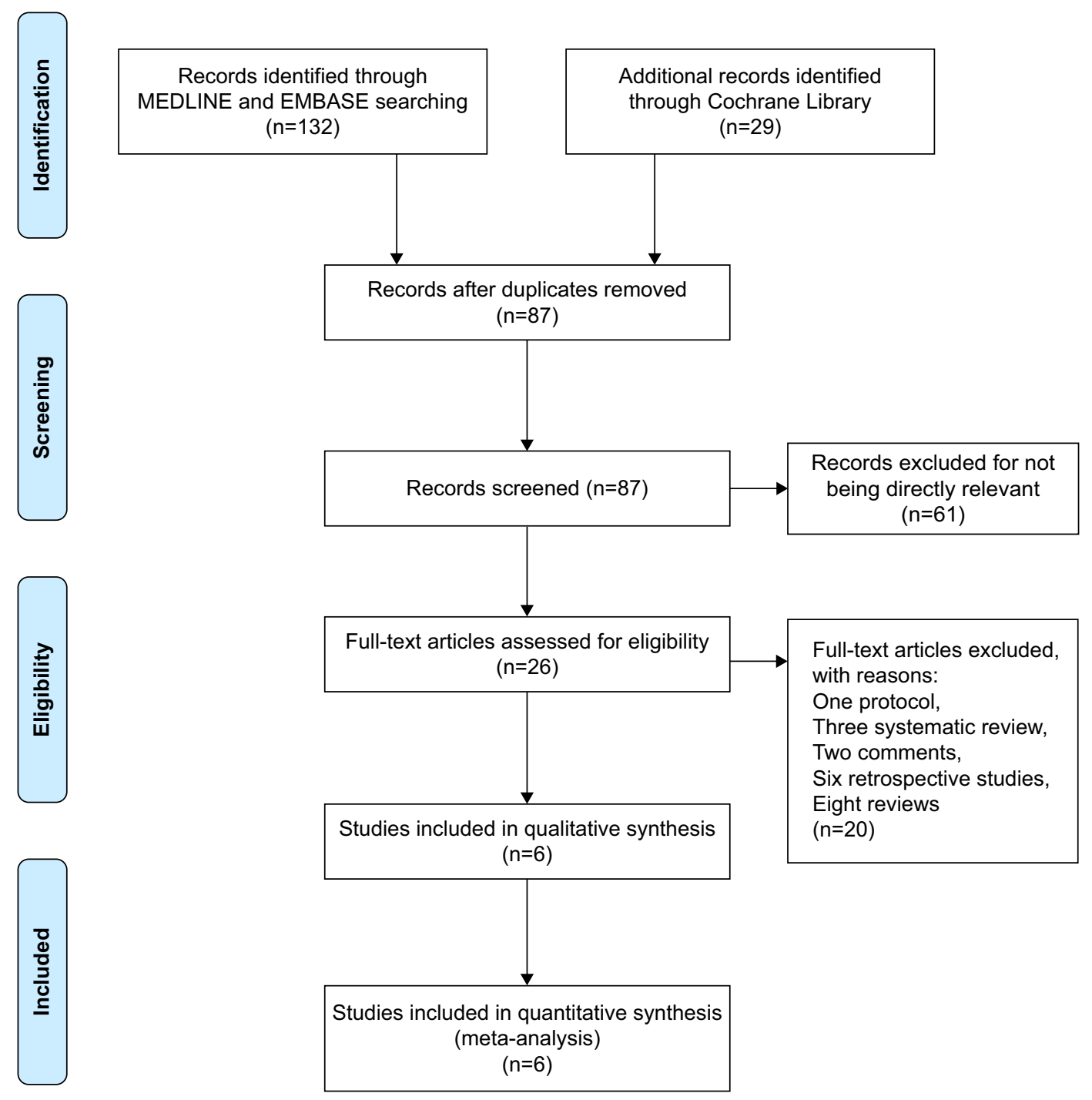

Figure I The study search, selection, and inclusion process. 
Figure 2B), or BI response (RR 0.95, 95\% CI 0.84-1.08, $P=0.44$; Figure $2 \mathrm{C}$ ). In the safety analysis, cerebrolysin did not increase the risk of adverse events (RR 0.98, 95\% CI $0.88-1.09, P=0.67$; Figure 3A), risk of serious adverse events (RR $1.20,95 \%$ CI $0.86-1.66, P=0.29$; Figure 3B), or the mortality rate (RR $0.86,95 \%$ CI $0.57-1.31$, $P=0.49$; Figure $3 \mathrm{C})$. The heterogeneity of the data ranged from 0 to $87 \%$ (Figures 2 and 3 ).

\section{Subgroup and sensitivity analysis}

The data extracted from the included studies were not sufficient to perform subgroup analyses to detect the influence of the cerebrolysin dosage, treatment duration, stroke severity at baseline, and follow-up time. Table 2 shows the outcomes of the follow-up time and baseline symptom severity subgroups.
Cerebrolysin showed a large advantage in patients with severe AIS. Sensitivity analysis detected that the Muresanu et $a l^{18}$ trial was the main source of heterogeneity due to the inclusion of only severe AIS patients in this trial.

\section{Quality of the included studies}

Full details regarding the risks of bias of the RCTs are shown in Figure 4. For allocation concealment, the risk of bias was unclear in four studies because the allocation scheme was not mentioned in the trials. For blinding of the outcome assessment, the risk of bias was unclear in three studies. For incomplete outcome data, the risk of bias was high in one study. For selective reporting, the risk of bias was unclear in one study. Except for these four items, no high or unclear risk of bias was observed in any of the other items.

A

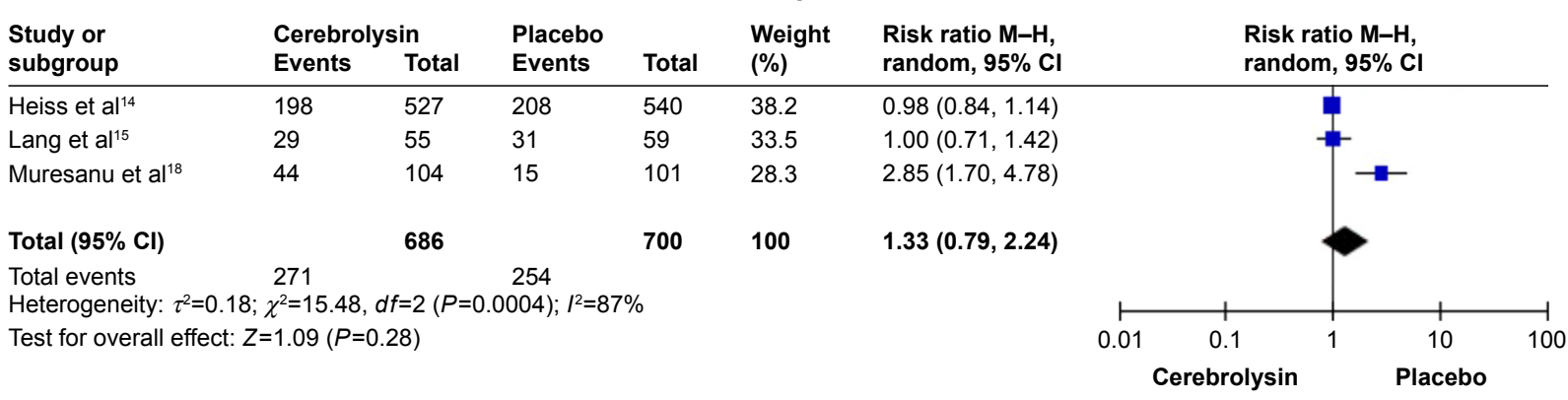

B

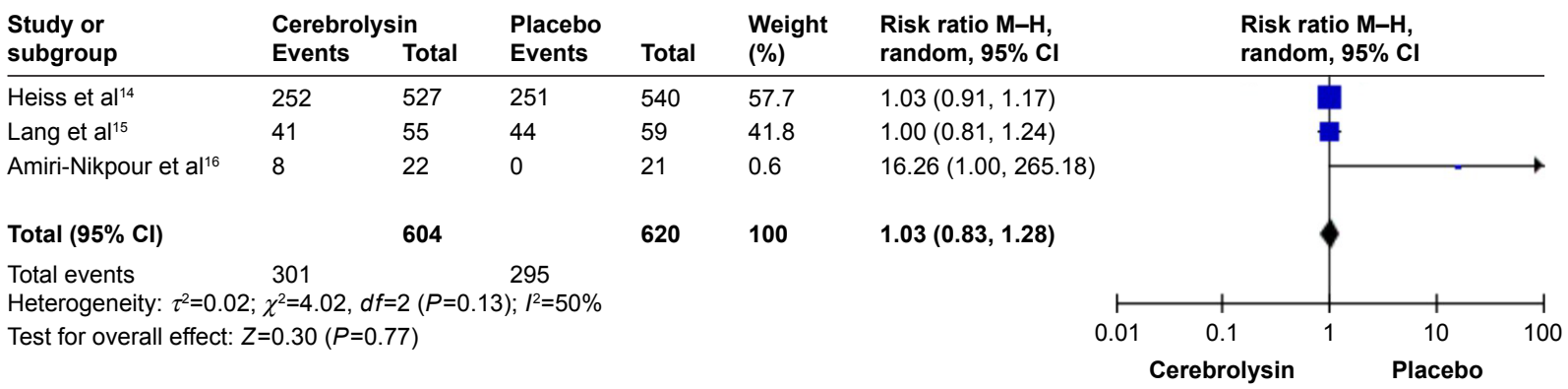

C

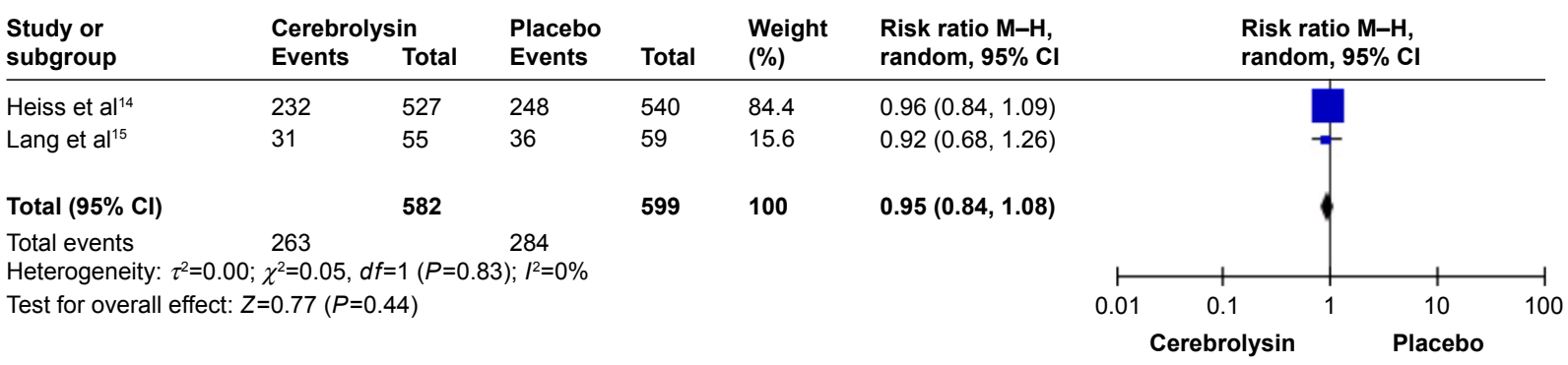

Figure 2 The pooled relative risk of the efficacy outcomes: (A) modified Rankin Scale (mRS) response, (B) National Institutes of Health Stroke Scale (NIHSS) response, and (C) Barthel Index (BI) response.

Notes: The diamonds indicate the estimated relative risk ( $95 \%$ confidence interval $[\mathrm{Cl}])$ for all patients together. The size of the squares indicates the sample size of the included studies.

Abbreviation: $\mathrm{M}-\mathrm{H}$, Mantel-Haenszel. 


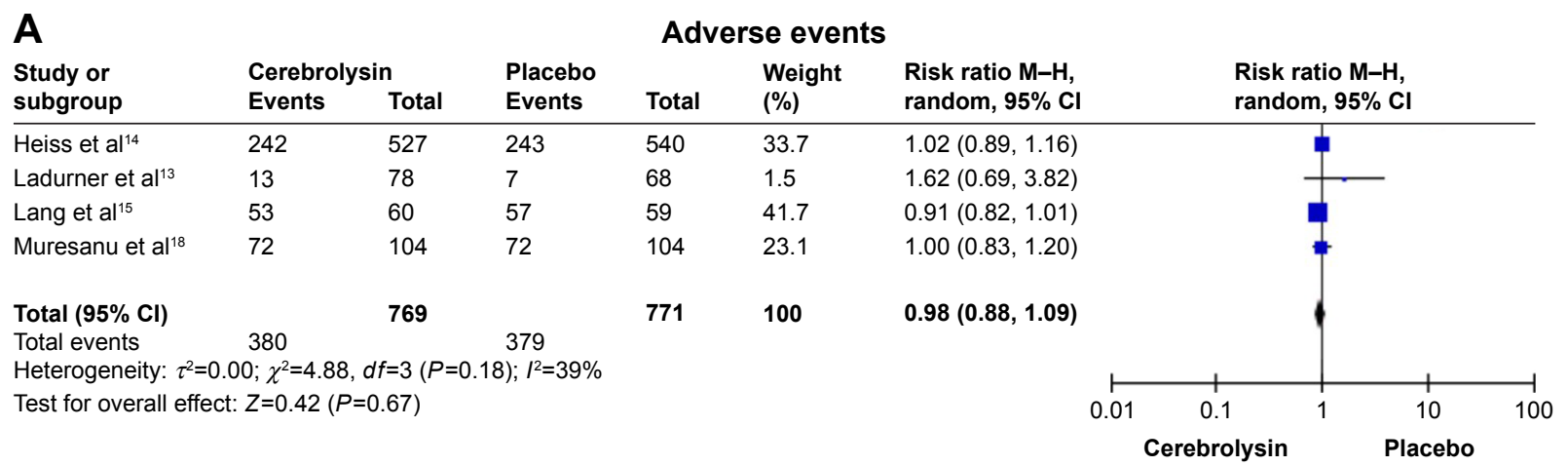

B

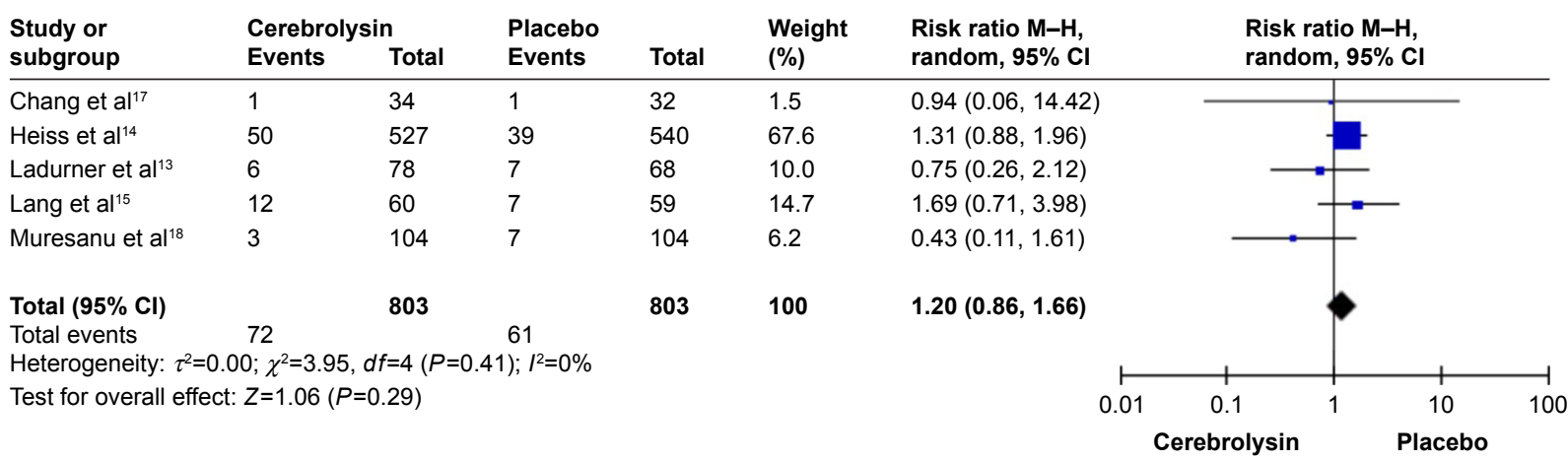

C

\begin{tabular}{|c|c|c|c|}
\hline $\begin{array}{l}\text { Study or } \\
\text { subgroup }\end{array}$ & $\begin{array}{l}\text { Cerebrolysin } \\
\text { Events }\end{array}$ & Total & $\begin{array}{l}\text { Placebo } \\
\text { Events }\end{array}$ \\
\hline Chang et $\mathrm{al}^{17}$ & 0 & 34 & 0 \\
\hline Heiss et $\mathrm{al}^{14}$ & 28 & 527 & 32 \\
\hline Ladurner et $\mathrm{al}^{13}$ & 6 & 78 & 6 \\
\hline Lang et $\mathrm{al}^{15}$ & 4 & 60 & 4 \\
\hline Muresanu et $a^{18}$ & 0 & 104 & 4 \\
\hline \multicolumn{2}{|l|}{ Total $(95 \% \mathrm{Cl})$} & \multicolumn{2}{|l|}{803} \\
\hline Total events & 38 & & 46 \\
\hline \multicolumn{4}{|c|}{ Heterogeneity: $\tau^{2}=0.00 ; \chi^{2}=2.01, d f=3(P=0.57) ; l^{2}=0 \%$} \\
\hline \multicolumn{4}{|c|}{ Test for overall effect: $Z=0.69(P=0.49)$} \\
\hline
\end{tabular}

Mortality rate

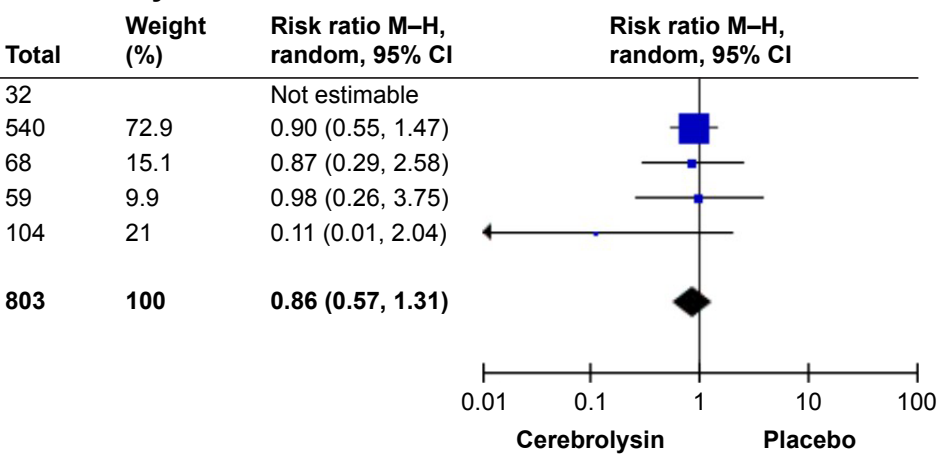

Figure 3 The pooled relative risk of the safety outcomes: $(\mathbf{A})$ adverse events, $(\mathbf{B})$ serious adverse events, and $(\mathbf{C})$ mortality rate.

Notes: The diamonds indicate the estimated relative risk $(95 \%$ confidence interval $[\mathrm{Cl}])$ for all patients together. The size of the squares indicates the sample size of the included studies.

Abbreviation: M-H, Mantel-Haenszel.

\section{Discussion}

Treatment with cerebrolysin for functional recovery in patients with AIS might be questionable based on the evidence of the present meta-analysis. We found that cerebrolysin treatment showed no advantage in global neurologic recovery as assessed using the mRS, NIHSS, and BI. A subgroup analysis indicated that cerebrolysin showed a superiority in patients with severe AIS. Cerebrolysin showed a satisfactory safety performance in that it did not increase the incidence of adverse events, serious adverse events, or mortality. This evidence was based on the limited data from the included RCTs.
Cerebrolysin has been regarded as an ideal agent for functional recovery after AIS because of its dual attributes including neuroprotective properties and neurotrophic activity. ${ }^{6}$ Neuroprotective therapies could be used to intervene at various stages of the ischemic pathophysiological cascade and could probably have a superior clinical application. ${ }^{21}$ However, all clinical trials available to date attempting to confirm the experimental observations of the neuroprotective effects of calcium channel blockers, N-methyl-D-aspartate antagonists, glutamate release inhibitors, potassium channel agonists, and radical scavengers have delivered disappointing results. ${ }^{22,23}$ Neurotrophic molecules have been considered 
Table 2 Subgroup analysis of follow-up time and symptom severity at baseline

\begin{tabular}{|c|c|c|c|c|c|c|c|c|}
\hline \multirow[t]{3}{*}{ Trials } & \multicolumn{4}{|l|}{ Follow-up time } & \multicolumn{4}{|c|}{ Symptom severity at baseline } \\
\hline & \multicolumn{2}{|l|}{ Middle point } & \multicolumn{2}{|l|}{ End point } & \multicolumn{2}{|c|}{$\begin{array}{l}\text { Mild-moderate } \\
\text { symptoms }\end{array}$} & \multicolumn{2}{|l|}{ Severe symptoms } \\
\hline & Outcomes & $P$-value & Outcomes & $P$-value & Outcomes & $P$-value & Outcomes & $P$-value \\
\hline \multirow[t]{2}{*}{ Muresanu et al ${ }^{18}$} & ARAT scale & $<0.0001$ & ARAT scale & $<0.0001$ & N/A & N/A & ARAT scale & $<0.0001$ \\
\hline & & & $\begin{array}{l}\text { I2 different outcome } \\
\text { scales on global status }\end{array}$ & $<0.0001$ & & & $\begin{array}{l}\text { I2 different outcome } \\
\text { scales on global status }\end{array}$ & $<0.0001$ \\
\hline \multirow[t]{5}{*}{ Chang et $\mathrm{al}^{17}$} & FMA scale & $>0.05$ & FMA scale & $>0.05$ & FMA scale & $>0.05$ & FMA scale & $<0.05$ \\
\hline & Fractional anisotropy & $>0.05$ & Fractional anisotropy & $>0.05$ & & & & \\
\hline & Axial diffusivity & $>0.05$ & Axial diffusivity & $<0.01$ & & & & \\
\hline & Radial diffusivity & $>0.05$ & Radial diffusivity & $<0.01$ & & & & \\
\hline & rsfMRI & $>0.05$ & rsfMRI & $>0.05$ & & & & \\
\hline \multirow[t]{7}{*}{ Amiri-Nikpour et al ${ }^{16}$} & NIHSS & 0.092 & NIHSS & 0.001 & $\mathrm{~N} / \mathrm{A}$ & N/A & $\mathrm{N} / \mathrm{A}$ & $\mathrm{N} / \mathrm{A}$ \\
\hline & Velocity of right MCA & 0.033 & Velocity of right MCA & 0.065 & & & & \\
\hline & Velocity of left MCA & 0.768 & Velocity of left MCA & 0.638 & & & & \\
\hline & Velocity of BA & 0.610 & Velocity of basilar BA & 0.657 & & & & \\
\hline & Right MCA PI & 0.010 & Right MCA PI & 0.014 & & & & \\
\hline & Left MCA PI & 0.286 & Left MCA PI & 0.277 & & & & \\
\hline & BA PI & 0.384 & BA PI & 0.828 & & & & \\
\hline \multirow[t]{4}{*}{ Lang et $\mathrm{al}^{15}$} & $\mathrm{mRS}$ & 0.366 & $\mathrm{mRS}$ & 0.984 & $\mathrm{~N} / \mathrm{A}$ & $\mathrm{N} / \mathrm{A}$ & $\mathrm{N} / \mathrm{A}$ & $N / A$ \\
\hline & NIHSS & 0.038 & NIHSS & 0.490 & & & & \\
\hline & $\mathrm{BI}$ & $0.84 I$ & $\mathrm{BI}$ & 0.673 & & & & \\
\hline & & & GOS scale & 0.882 & & & & \\
\hline \multirow[t]{4}{*}{ Heiss et $\mathrm{al}^{1 / 4}$} & - & - & $\mathrm{mRS}$ & $>0.05$ & $\mathrm{mRS}$ & $>0.05$ & $\mathrm{mRS}$ & 0.09 \\
\hline & & & NIHSS & $>0.05$ & NIHSS & $>0.05$ & NIHSS & 0.04 \\
\hline & & & $\mathrm{BI}$ & $>0.05$ & $\mathrm{BI}$ & $>0.05$ & & \\
\hline & & & SF-I2 & $>0.05$ & & & & \\
\hline \multirow[t]{7}{*}{ Ladurner et $\mathrm{al}^{13}$} & CNS section AI & $<0.05$ & CNS & $>0.05$ & $\mathrm{~N} / \mathrm{A}$ & $\mathrm{N} / \mathrm{A}$ & $N / A$ & $N / A$ \\
\hline & MMSE & $<0.05$ & CNS section AI & $>0.05$ & & & & \\
\hline & SST & $<0.05$ & $\mathrm{BI}$ & $>0.05$ & & & & \\
\hline & & & GCS & $>0.05$ & & & & \\
\hline & & & CGI & $>0.05$ & & & & \\
\hline & & & MMSE & $>0.05$ & & & & \\
\hline & & & SST & $<0.05$ & & & & \\
\hline
\end{tabular}

Abbreviations: ARAT, Action Research Arm Test; BA, basilar artery; BI, Barthel index; CGI, clinical global impression; CNS, Canadian Neurological Scale; FMA, Fugl-Meyer Assessment; GCS, Glasgow Coma Scale; GOS, Glasgow Outcome Scale; MCA, middle cerebral artery; MMSE, Mini-Mental State Examination; mRS, modified Rankin Scale; N/A, not applicable; NIHSS, National Institutes of Health Stroke Scale; PI, pulsatility index; rsfMRI, resting-state functional magnetic resonance imaging; SF-I2, short form 12 items; SST, Syndrome Short Test.

as new strategies for treatment and prevention of ischemic strokes. ${ }^{24}$ Clinical trials of neurotrophic substances have not obtained the expected results of infarct size reduction and improved behavioral outcomes in animal models. ${ }^{25}$ Cerebrolysin is the only multitargeted drug with effects on multiple pathophysiologic events. ${ }^{26}$ Preclinical studies have shown consistent results of positive effects of cerebrolysin on functional recovery after AIS through multiple pathophysiological pathways. ${ }^{7-10}$ The translational step to move the experimental data from bench to bedside has been performed under the guidance of the Stroke Therapy Academic Industry Roundtable. ${ }^{27}$

The present meta-analysis showed negative consolidated results from the included RCTs. Cerebrolysin had no advantage in global neurologic recovery as assessed using the mRS, NIHSS, and BI. Further subgroup analysis indicated that cerebrolysin showed a superiority in patients with severe AIS. The cerebral autoregulatory mechanism made the spontaneous recovery of patients with AIS possible, especially in those with mild ischemic stroke. ${ }^{28} \mathrm{~A}$ high rate of spontaneous recovery under placebo might reduce the superiority of the cerebrolysin treatment arm. The Lang et al trial recruited a large number of patients with mild strokes, which observed an excellent outcome ( $\mathrm{mRS} 0$ or 1 ) in approximately $53 \%$ of the patients from both the cerebrolysin and placebo groups. ${ }^{15}$ These results were superior to the findings of previous studies in which only approximately $40 \%$ of the patients showed a favorable recovery in the placebo group..$^{29,30}$ 


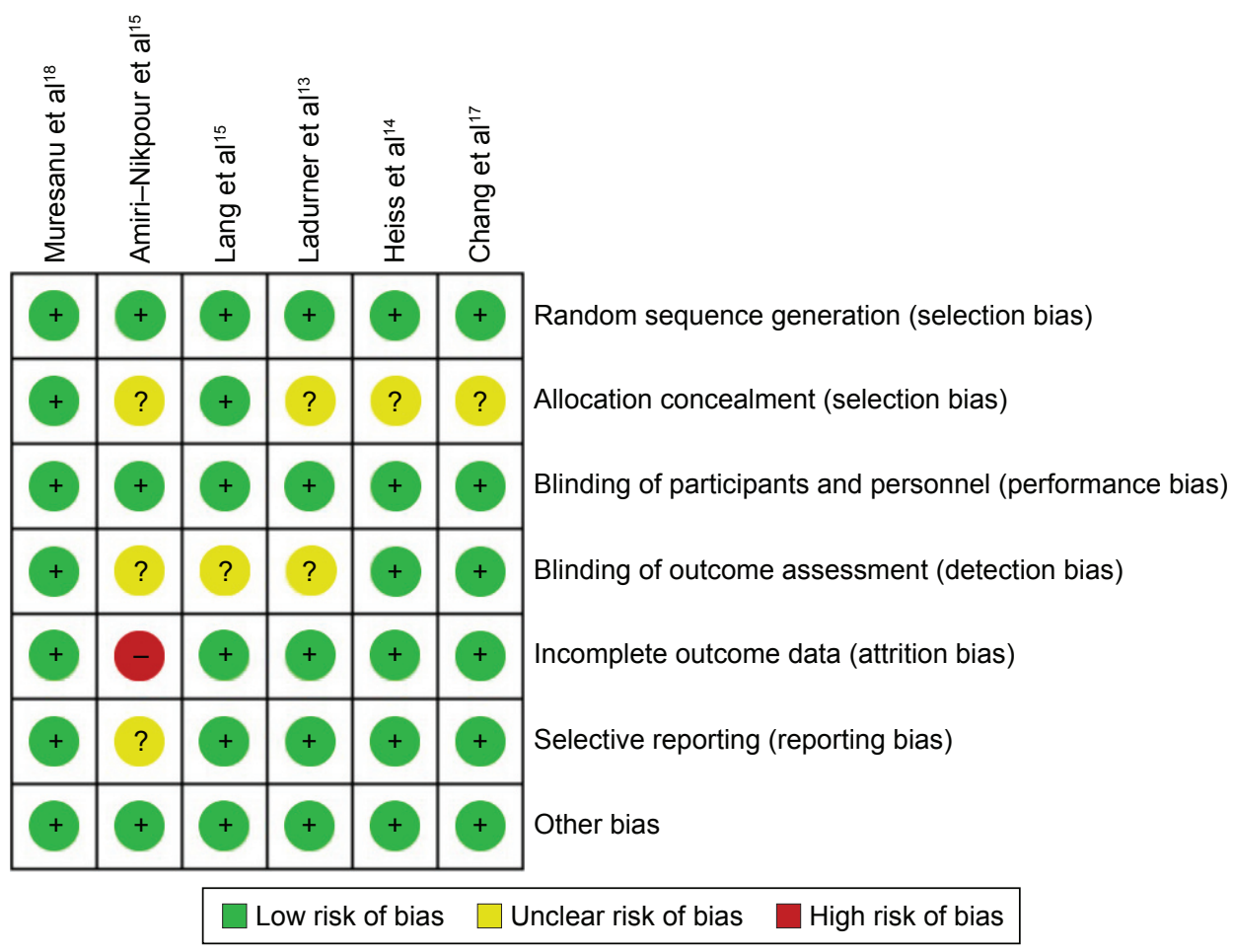

Figure 4 Risk of bias: a summary table for each risk-of-bias item for each study.

The Ladurner et al trial also included a group of patients with a mild pattern of baseline ischemic impairment. ${ }^{13}$ The average neurological score of the two groups was close to the maximum test score. ${ }^{13}$ The pronounced ceiling effect impedes a measurable treatment effect for the test substance. ${ }^{13}$ However, the Amiri-Nikpour et al trial showed a sizable advantage of cerebrolysin in the NIHSS response, although this trial included several AIS patients with mild symptoms at baseline. ${ }^{16}$ The unexpected mildly affected patients in these studies might be the main cause of the inconclusive results. In the subgroup of AIS patients with severe neurological deficits at baseline, cerebrolysin treatment showed a superiority compared with the effect of placebo in three RCTs. ${ }^{14,17,18} \mathrm{We}$ could not perform a subgroup analysis to detect the influence of stroke severity based on the NIHSS scores at baseline due to the insufficient data from the included studies. Further large RCTs should examine the value of cerebrolysin in a subgroup of patients with a baseline NIHSS of 12 points.

The failure of cerebrolysin treatment to translate from experimental studies to clinical application might be due in part to the lack of consideration paid to the limited time window in the pathophysiological cascade. ${ }^{31}$ Previous results from neuroprotective therapy have indicated that early intervention within the first few hours after stroke might achieve favorable outcomes. ${ }^{21}$ Cerebrolysin was regarded to achieve effects even if the treatment was initiated with a delay of 48 hours after stroke onset in animal studies. ${ }^{7,12}$ This appears to be a wide therapeutic time window when compared to that of other neuroprotective substances. ${ }^{13}$ The therapeutic time window of the included trials ranged from 6 hours to 7 days after stroke onset. Due to the limited number of included studies, a subgroup analysis was unfit for detecting the influence of different therapeutic time windows. The Amiri-Nikpour et al trial administered cerebrolysin or placebo within 6 hours after arriving to the hospital, which showed significant improvements in the neurological outcomes and the pulsatility index of the middle cerebral artery at Day $90 .{ }^{16}$ Similar functional recovery was observed in the Lang et al trial that delivered cerebrolysin within 1 hour after thrombolytic therapy with recombinant tissue plasminogen activator. ${ }^{15}$ A therapeutic time delay over 6 hours seemed to weaken the efficacy of cerebrolysin on the neurologic functional recovery according to the negative findings of the trials of Ladurner et al, Heiss et al, and Chang et al..$^{13,14,17}$ Although the remodeling of neural connectivity and the remapping of new structural and functional circuits after AIS requires a long time, ${ }^{32}$ early intervention with cerebrolysin within 6 hours might achieve a favorable clinical outcome.

All of the included trials showed accelerated recovery at early time points of assessment. ${ }^{13-18}$ However, these inspiring results vanished at the final visit on Day 90 in several 
trials. ${ }^{13,15,16}$ The Ladurner et al trial revealed a significant benefit in the cerebrolysin group at Days 1, 3, 7, 14, and 21, but no effects were observed at the final visit on Day $90 .{ }^{13}$ A difference between the cerebrolysin and placebo groups was observed at Day 2, and this difference achieved a peak with an NIHSS response rate of $72.2 \%$ for the cerebrolysin group vs $50.8 \%$ for the placebo group at the end of the treatment on Day 10 in the Lang et al trial. ${ }^{15}$ This positive effect was maintained until Day 30 but vanished at the 3-month follow-up investigation. ${ }^{15}$ Similar results were observed in the Amiri-Nikpour et al trial. ${ }^{16}$ The hypothesis that cerebrolysin had neuroprotective effects during the treatment period should be considered to account for these data. However, the present meta-analysis could not test this hypothesis due to the limited data from the included trials. Further studies should examine the effect of cerebrolysin in a long treatment period.

Several limitations should be noted in this meta-analysis. Only six published RCTs with 1,649 patients were involved in the final analysis. This might cause publication bias, although the Begg-Mazumdar rank correlation test yielded negative results (data not shown). The included studies exhibited spotty quality, which is shown in Figure 4. Investigators should be careful in further clinical practice. In addition, the present meta-analysis could not detect the potential effects of the combined use of thrombolytic drugs, aspirin prevention, and rehabilitation therapy.

\section{Conclusion}

Cerebrolysin has no significant efficacy on the neurological functional recovery in patients with AIS. Routine administration of cerebrolysin to patients with AIS cannot be supported by the available evidence from RCTs. Further studies should focus on the efficacy of cerebrolysin administered within 6 hours after AIS and maintained for a longer period in patients with severe symptoms.

\section{Acknowledgments}

This study was supported by grants from the National Natural Science Foundation of China (No 81171096 and No 81371433) to JMZ.

\section{Disclosure}

The authors report no conflicts of interest in this work.

\section{References}

1. Murray CJ, Lopez AD. Measuring the global burden of disease. N Engl J Med. 2013;369(5):448-457.

2. Berkhemer OA, Fransen PS, Beumer D, et al; MR CLEAN Investigators. A randomized trial of intraarterial treatment for acute ischemic stroke. N Engl J Med. 2015;372(1):11-20.
3. Schmitz ML, Simonsen CZ, Hundborg H, et al. Acute ischemic stroke and long-term outcome after thrombolysis: nationwide propensity score-matched follow-up study. Stroke. 2014;45(10):3070-3072.

4. Zahuranec DB, Majersik JJ. Percentage of acute stroke patients eligible for endovascular treatment. Neurology. 2012;79(13 Suppl 1): S22-S25.

5. Stinear C. Prediction of recovery of motor function after stroke. Lancet Neurol. 2010;9(12):1228-1232.

6. Masliah E, Diez-Tejedor E. The pharmacology of neurotrophic treatment with cerebrolysin: brain protection and repair to counteract pathologies of acute and chronic neurological disorders. Drugs Today (Barc). 2012;48 (Suppl A):3-24.

7. Gutmann B, Hutter-Paier B, Skofitsch G, Windisch M, Gmeinbauer R. In vitro models of brain ischemia: the peptidergic drug cerebrolysin protects cultured chick cortical neurons from cell death. Neurotox Res. 2002;4(1):59-65.

8. Riley C, Hutter-Paier B, Windisch M, Doppler E, Moessler H, Wronski R. A peptide preparation protects cells in organotypic brain slices against cell death after glutamate intoxication. J Neural Transm (Vienna). 2006;113(1):103-110

9. Hartbauer M, Hutter-Paier B, Skofitsch G, Windisch M. Antiapoptotic effects of the peptidergic drug cerebrolysin on primary cultures of embryonic chick cortical neurons. J Neural Transm (Vienna). 2001; 108(4):459-473.

10. Zhang L, Chopp M, Meier DH, et al. Sonic hedgehog signaling pathway mediates cerebrolysin-improved neurological function after stroke. Stroke. 2013;44(7):1965-1972.

11. Zhang C, Chopp M, Cui Y, et al. Cerebrolysin enhances neurogenesis in the ischemic brain and improves functional outcome after stroke. J Neurosci Res. 2010;88(15):3275-3281.

12. Ren J, Sietsma D, Qiu S, Moessler H, Finklestein SP. Cerebrolysin enhances functional recovery following focal cerebral infarction in rats. Restor Neurol Neurosci. 2007;25(1):25-31.

13. Ladurner G, Kalvach P, Moessler H; Cerebrolysin Study Group. Neuroprotective treatment with cerebrolysin in patients with acute stroke: a randomised controlled trial. J Neural Transm (Vienna). 2005; 112(3):415-428

14. Heiss WD, Brainin M, Bornstein NM, Tuomilehto J, Hong Z; Cerebrolysin Acute Stroke Treatment in Asia (CASTA) Investigators. Cerebrolysin in patients with acute ischemic stroke in Asia: results of a double-blind, placebo-controlled randomized trial. Stroke. 2012; 43(3):630-636.

15. Lang W, Stadler CH, Poljakovic Z, Fleet D; Lyse Study Group. A prospective, randomized, placebo-controlled, double-blind trial about safety and efficacy of combined treatment with alteplase (rt-PA) and cerebrolysin in acute ischaemic hemispheric stroke. Int J Stroke. 2013;8(2):95-104.

16. Amiri-Nikpour MR, Nazarbaghi S, Ahmadi-Salmasi B, Mokari T, Tahamtan U, Rezaei Y. Cerebrolysin effects on neurological outcomes and cerebral blood flow in acute ischemic stroke. Neuropsychiatr Dis Treat. 2014;10:2299-2306.

17. Chang WH, Park CH, Kim DY, et al. Cerebrolysin combined with rehabilitation promotes motor recovery in patients with severe motor impairment after stroke. BMC Neurol. 2016;16:31.

18. Muresanu DF, Heiss WD, Hoemberg V, et al. Cerebrolysin and Recovery After Stroke (CARS): a randomized, placebo-controlled, double-blind, multicenter trial. Stroke. 2016;47(1):151-159.

19. Moher D, Liberati A, Tetzlaff J, Altman DG; PRISMA Group. Preferred reporting items for systematic reviews and meta-analyses: the PRISMA statement. J Clin Epidemiol. 2009;62(10):1006-1012.

20. Higgins JP, Altman DG, Gøtzsche PC, et al; Cochrane Bias Methods Group; Cochrane Statistical Methods Group. The Cochrane Collaboration's tool for assessing risk of bias in randomised trials. $B M J$. 2011;343:d5928.

21. Tuttolomondo A, Pecoraro R, Arnao V, Maugeri R, Iacopino DG, Pinto A. Developing drug strategies for the neuroprotective treatment of acute ischemic stroke. Expert Rev Neurother. 2015;15(11):1271-1284. 
22. Ginsberg MD. Neuroprotection for ischemic stroke: past, present and future. Neuropharmacology. 2008;55(3):363-389.

23. Lees KR. Advances in neuroprotection trials. Eur Neurol. 2001; 45(1):6-10.

24. Abe K. Therapeutic potential of neurotrophic factors and neural stem cells against ischemic brain injury. J Cereb Blood Flow Metab. 2000;20(10):1393-1408.

25. Kalluri HS, Dempsey RJ. Growth factors, stem cells, and stroke. Neurosurg Focus. 2008;24(3-4):E14.

26. Bornstein N, Poon WS. Accelerated recovery from acute brain injuries: clinical efficacy of neurotrophic treatment in stroke and traumatic brain injuries. Drugs Today (Barc). 2012;48 (Suppl A):43-61.

27. Fisher M, Albers GW, Donnan GA, et al; Stroke Therapy Academic Industry Roundtable IV. Enhancing the development and approval of acute stroke therapies: Stroke Therapy Academic Industry roundtable. Stroke. 2005;36(8):1808-1813.
28. Heyer EJ, Mergeche JL, Connolly ES Jr. Middle cerebral artery pulsatility index and cognitive improvement after carotid endarterectomy for symptomatic stenosis. J Neurosurg. 2014;120(1):126-131.

29. Wahlgren N, Ahmed N, Dávalos A, et al; SITS investigators. Thrombolysis with alteplase 3-4.5 h after acute ischaemic stroke (SITS-ISTR): an observational study. Lancet. 2008;372(9646):1303-1309.

30. Tissue plasminogen activator for acute ischemic stroke. The National Institute of Neurological Disorders and Stroke rt-PA Stroke Study Group. N Engl J Med. 1995;333(24):1581-1587.

31. Hossmann KA. The two pathophysiologies of focal brain ischemia: implications for translational stroke research. J Cereb Blood Flow Metab. 2012;32(7):1310-1316.

32. Murphy TH, Corbett D. Plasticity during stroke recovery: from synapse to behaviour. Nat Rev Neurosci. 2009;10(12):861-872.

\section{Publish your work in this journal}

Drug Design, Development and Therapy is an international, peerreviewed open-access journal that spans the spectrum of drug design and development through to clinical applications. Clinical outcomes, patient safety, and programs for the development and effective, safe, and sustained use of medicines are the features of the journal, which has also been accepted for indexing on PubMed Central. The manuscript management system is completely online and includes a very quick and fair peer-review system, which is all easy to use. Visit http://www.dovepress.com/testimonials.php to read real quotes from published authors.

Submit your manuscript here: http://www.dovepress.com/drug-design-development-and-therapy-journal 